\title{
A different perspective towards urban planning based on GIS and VGI techniques
}

\author{
Peshawa M.M. Karash \\ Geomatics Engineer:Mitta AB, Stockholm, Sweden, peshawa.karrash@gmail.com
}

\begin{abstract}
Urban planning is considered one of the most critical topics in the design and development strategies of cities. There are a vast amount of urban planning rule sets that focus on dominating the geometrical perspective on other approaches when it comes to planning decisions. In this paper, a different point of view towards urban planning is suggested concentrating on the topological analysis and relation between city elements. Streets of cities are chosen to reflect this topological relation and to investigate the topological relations to the limits; six different sized cities were selected for analysis; three large cities and three relatively small ones. Results of study uncovered the hierarchical pattern underlying in street structure of cities; analyses showed that street networks in large cities have a higher degree of hierarchical level than in relatively small cities. Urban planners can get benefit from the results of analyses in this study to make better planning decisions in large or small cities. The paper provides analysis results towards achieving the optimum goals of urban planning to make cities more living and more efficient.
\end{abstract}

Keywords: Geometric analysis, Topological analysis, Head/tail breaks, scale-free property, Small- world property.

Received: March $07^{\text {th }}, 2021 /$ Accepted: June $28^{\text {th }}, 2021 /$ Online: June $30^{\text {th }}, 2021$

\section{Introduction}

When it comes to designing new cities or densifying old ones, an efficient engineering strategy must be implemented for transforming cities in to more sustainable ones. However, without a rigid urban planning scheme, there will be serious consequences on the living environment in the cities. It is only by comprehensive planning strategies, a living and sustainable cities can be attained. In contrast to that, noticeable setbacks are realized in the efficiency of cities when implying poor planning, meaning that when the spaces between city objects and composers are not perfectly utilized; one way to help with better planning is performing spatial analysis. (LeGates et al., 2009) stated in their research that city planners should master spatial thinking skills and spatial analysis.

Spatial analysis is the key factor in urban planning for uncovering the hierarchical patterns within cities. Spatial analysis can been defined as a technique to examine, reveal, and clarifying spatial phenomena occurring on the surface of the earth (Murayama \& Thapa, 2011). By making a spatial analysis on city composers' better future planning decisions can be made. The analysis is performed on various sources of spatial data that has been gathered using surveying instruments, remote sensing techniques, satellite images, Global Positioning Systems (GPS), and so forth. Remote sensing and Geographic Information Systems (GIS) techniques have been increasingly utilized to facilitate urban planning and development (Jianquan \& Masser, 2001). 
In this study, GIS was utilized as the main software to analyze and visualize the data. Geographic information systems (GIS) are computer-based systems that make it easier to deal with georefrenced data in terms of data input, storing, and manipulation (Hussain, 2016). Street networks within the cities can be used as a suitable data source to carry out spatial analysis for the purposes mentioned above, in this study, street networks extracted from Open Street Maps (OSM) was used is the main data source which belongs to Volunteered Geographic Information (VGI) package. VGI is voluntarily distributed by individuals; it includes geographic data creating, assembling and disseminating (Goodchild, 2007) and is widely used now. OSM provides such freely edited and used map data and it offers the way to collect data for specific studies that make it be a most useful way for customers (Haklay \& Weber, 2008). Some researchers had analyzed the credibility of VGI. A comparative study showed that the quality of VGI could be treated as reaching a good level and the participants that distribute VGI are diligent and committed (Haklay, 2010).

Due to the fact that in every city there are some streets which are highly connected and there are others which have fewer connections, so by showing this hierarchy the underlying pattern of cities could be uncovered. Streets with high connectivity attract vast amount of people and vehicles at any time of the year, such streets are considered to be more crowded and having much more noise as compared to other less connective streets. Moreover, highly connected streets within the street network in a city are considered most important streets in the network. In this study, spatial analysis is focused on the streets in cities to uncover the underlying hierarchical pattern of sampled cities.

To accomplish the task, streets have to be analyzed in such a way that the connection between every single street is known relative to other streets. In other words, the relation between streets shall be perceived. This kind of analysis that focuses on the relation between objects is called topological analysis which is different from the geometrical one which focuses on the geometry of objects not the relation between them. Axial lines which are defined as the longest visibility lines crossing each other and running through open space of an urban environment (Hillier \& Hanson, 1984) can be used as a tool for representing the streets. It is worth mentioning that axial lines may not be the best method to represent phenomena which occur in the city because it has some significant drawbacks. The axial representation has been questioned by researchers for its validity, as it is neither cognitively sound nor computationally operable (Jiang \& Claramunt, 2002). For these reasons, several attempts were made to find alternatives to axial lines, some of these attempts were discovering of named streets and natural streets.

At a Geographic Information Systems (GIS) database level, streets are merged with street segments which belong to the same name - named streets (Jiang \& Claramunt, 2004), or streets are naturally merged with other street segments that form good continuity-natural streets (Thomson, 2003). Therefore, it can be stated that named streets and natural streets constitute the street-based topological representations to be used as sufficient data for making spatial analysis on urban street networks. As it is obvious streets are not isolated objects, but connected and crossing each other. This relation between streets enables us to perceive the hierarchies of street networks. According to (Jiang \& Liu, 2009) three main algorithms must be followed In order to create street-based topology; firstly, street segments are connected based on Gestalt principle of good continuity. Secondly, segments without names should be merged into neighboring segments and then merge all segments according to unique names. Lastly, isolated streets or lines should be identified and excluded from forming a topology.

This study focuses on spatial analysis performed on street networks to understand the street structure and uncover the underlying hierarchical pattern of selected cities. Through the process of attaining the results, theoretical concepts and practical procedures will be highlighted. The remainder of this paper is composed of four parts. In section 2, materials and methods of this research is introduced and explained in detail. In section 3, data processing steps are highlighted to familiarize the reader with the exact procedure done to accomplish the task. In section 4 , the results of the research are discussed. Finally, in section 5, the conclusion and the results of the study is presented. 


\section{Materials and methods}

Cities are basically composed of streets, buildings, and spaces between them. Mapping the streets is considered to be one of the most effective methods to represent the cities and uncover underlying structural pattern of each city. It has to be noted that most of the phenomena around the individual in the real world are occurring in a non-homogeneous pattern, for example, there are many small events than large ones and there are many short buildings than taller ones and so forth. These phenomena of unequal arrangement and distribution are called power law, which can be seen in streets also as there are more short streets than the large one. There are many terms to refer to the power laws in the literature, such as scaling, scale invariance, scale-free, universality, hierarchy, heterogeneity, and non-linearity. Research on the origins of power laws and efforts to observe and validate them in the real world are particularly active in many fields of science including, for instance, physics, economics, biology and more recently, computer science (Mitzenmacher, 2004). To confirm this notion Jiang (2007) made a practical example in which 40 US cities were chosen as samples, he discovered that about $20 \%$ of streets of the network have lengths greater than average while $80 \%$ of streets have lengths less than average value. Scaling and topology are considered the key factors for spatial analysis.

Topology demonstrates how objected are related to each other, in topological representation, the geometry of objects may be distorted but the main purpose of the map will be retained and showed more simply. Existing geographic representations, such as raster and vector, are essentially geometry-based because of involved locations, directions, and sizes. These representations are mainly driven by a static map metaphor (Yuan et al. 2005; Goodchild et al. 2007) for representing and characterizing the Earth's surface. It is only by preserving the true link between objects; correct navigation and measurements could be performed, a geographer can draw a topographical map of within a country, a student in school can tell where exactly a specific phenomenon takes place and so on. By losing geometric representation maps will not very beneficial and maybe systems such as GPS will not be required at all, this explains why Tobler's law, everything is related to everything else but near things are more related than distant things, is considered first law of geography. In one hand, Geometry-based representations fail to capture the spatial property of heterogeneity across all scales (Jiang et al., 2008). Simplicity in representation and homogeneity are important characteristics of this way of representation. However, availability in only one scale remains as a significant drawback of this kind of representation due to the fact that most of the real-world features are multi-scaled objects. Moreover, it is essential to mention that Gaussian's way of thinking is dominant in this kind of representation so maps tend to be linear, equilibrium and static.

On the other hand, Topology-based representations basically developed to show the structure of the map from the perspective of topology and wholeness. The topology among meaningful geographic features, such as streets, cities, buildings, mountains, and rivers, because the topology enables us to see the underlying scaling property or hierarchy (Jiang, 2018). An example of such representation can be cartograms, in which geometrical shapes are distorted but underlying topological relationships are retained. An example of cartogram map could be the London Underground map, it clearly shows the underlying structure as a transportation map, despite that the geometric relations between locations shown in map dramatically distorted, but one can easily use it to find his/her initial station and decide on next desired station. It is worth mentioning that the stations are not in their true absolute locations but they are correct relative to their neighboring stations, which in turn serves the initial transportation purpose of that map.

Topology can perceived as an instrument that enables us to see the underlying spatial heterogeneity or scaling property of far more small things than large ones. The topology used in GIS, however, is essentially geometry based, referring to adjacent relationships of basic geometric elements such as points, lines, polygons, and pixels (Jiang, 2018). This type of representation is suitable for objects that available at multi-scale and which are characterized by the complex structure. An interesting fact about scaling property of far more small things than large ones is that this scaling property recursively repeating itself, and when it occurs more than twice it follows fractal thinking, this proves that such representation is fractal in thinking, which almost everything is 
fractal, not conventional Euclidean. Moreover, one should note that concepts of topology, scaling and beauty are directly proportional to each other, because only through scaling, beauty is obtained and without topology undoubtedly scaling will not have such impact on representations.

It has to be noted that components of city such roads, edges, nodes, lands, and buildings are related to each other. For example, edges will normally be the edges of districts and nodes will be meeting of paths to form the city as a whole. These extend the conventional geographical frame of reference to large- and small-scale spaces. Large-scale space is beyond human perception and cannot be perceived from a single vantage point; while small-scale space is presumably larger than the human body, but can be perceived from a single vantage point (Jiang et al., 2000). Space simply could be defined as the unlimited or incalculably great three dimensional realms or expanse in which all material and objects are located and all events occur. The notion of syntax, derived from linguistics, refers to relationships between different spaces, or interactions between space and society (Jiang \& Claramunt 2002). Configurational analyses represented by the method of syntax allows the modeling of a city that links intuition and science, and it can be used for designing and planning cities, as well as in research (Hillier, 2009).

Space Syntax method was developed by (Hillier et al., 1976) at the University College London, and it has been applied in urban studies. This method was originally used and tested in analyses of small settlements and buildings. Later on, computer programs were able to analyze complex spatial relationships within larger cities and regions. Space syntax also made it feasible to analyze how a street interrelates spatially to all other streets in a large city. In addition, space syntax allows the comparison of spatial configurative analyses in relation to social activities such as the flow of pedestrian movements through streets, land use patterns, or the distribution of crime (Hillier \& Sahbaz, 2005).

Simply syntax can be perceived as a relationship, so in order to understand the morphology of geographic space, it has to be broken into smaller parts and relationships between small parts are analyzed. Streets are used as links to connect small spaces. In addition to uncovering the hierarchical levels of urban streets, other spatial analysis can be made on the streets like small world and scale-free property, which further illustrates the underlying pattern of urban network and enables urban planners for a making better urban planning decisions for the future. Small-world property is obtained in a network when most nodes in the network, which are not neighbors, can be connected from every other with a small number of steps according to two measures; average path length and clustering coefficient. According to (Watts \& Strogatz, 1998) a graph could be examined to see the small world property when the average path length between nodes is very small and clustering coefficient is a high degree value. The scale-free property, which already explained in this paper, of a network is shown when the degree distribution of network fits power-law distribution.

In this study, streets networks from 6 different cities were chosen for the analysis. The selected cities were 3 relatively big cities (Erbil, Sulaimaniyah, and Kirkuk) and 3 realtevely small ones (Duhok, Halabja, and Koysinjaq) located in Kurdistan region of Iraq, different sized cities were intentionally selected to check different hierarchical levels in large and small cities (Figure 1). ARC GIS software with Axwoman extension was used to prepare the data and to carry out the analysis. Axwoman which is developed by Professor Bin Jiang and his colleagues provide powerful tools for creating natural streets from chopped polylines which represent individual streets. Pajek software was used to show if the selected cities follow a small world property by finding average path length and clustering coefficient in the network. MATLAB software was used check if the degree distribution of urban street network of each city fits power law distribution. Head/Tail breaks classification, explained in the next section, the method was applied to the street networks of cities to show the hierarchical level of each city. 


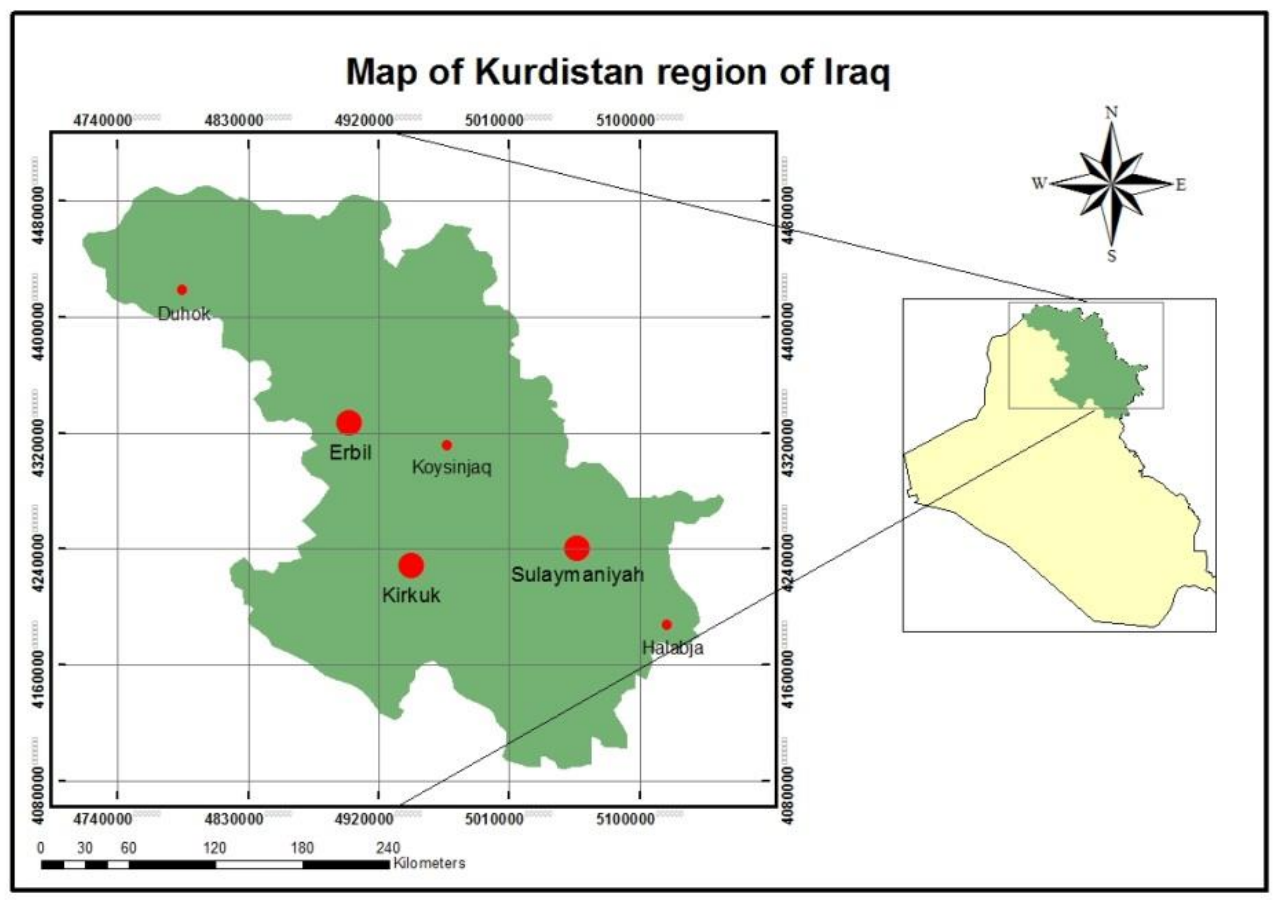

Figure 1. Map of Kurdistan region of Iraq showing cities of interest.

\section{Data Processing}

In this section a detailed procedure for applying spatial analysis on street networks in cities is explained, the purpose is to show the underlying structural pattern of cities. The first step was downloading the street network data from OSM website for each city of interest. GIS clip tool was used to crop the interesting areas within each city for further analysis; the coordinate system of downloaded data is WGS 1984 which has to be projected into a proper coordinate system to minimize the distortion. The projection which provides less distortion for this area is (UTM zone 38 north). A sample of a clipped and projected map of one of the cities in ARC GIS software is shown in (Figure 2).

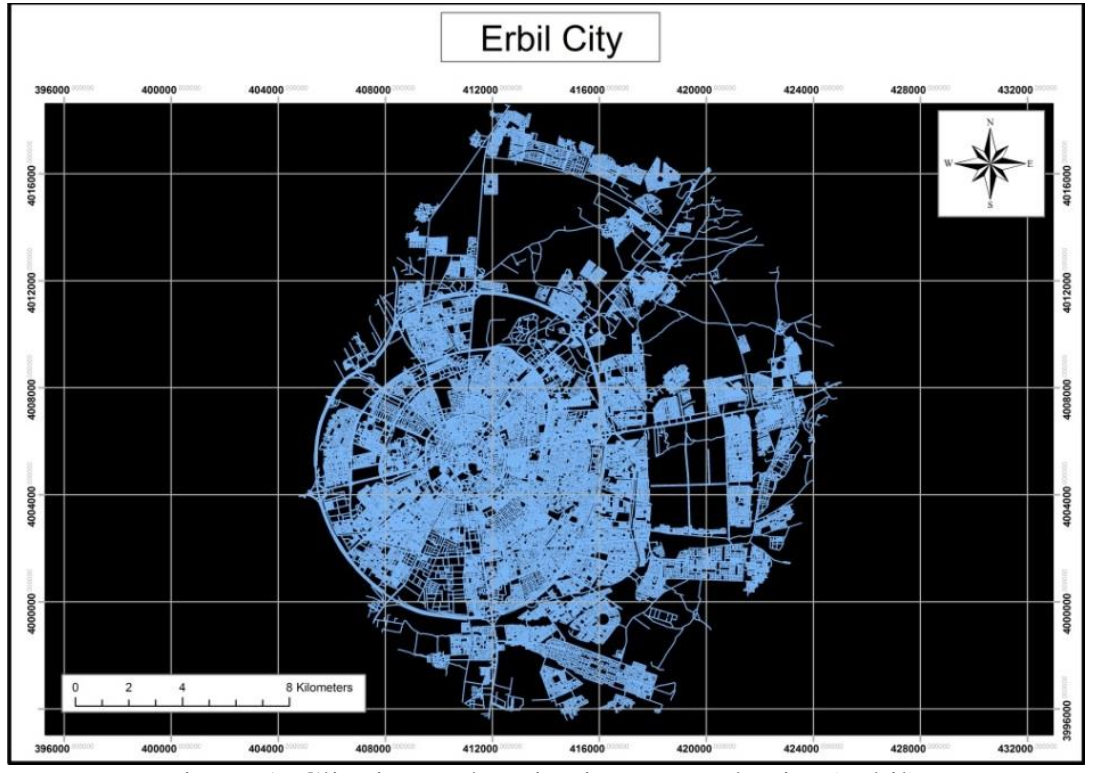

Figure 1. Clipping and projecting a sample city (Erbil). 
In the next step, isolated lines which may lead to stopping later processing were modified or deleted. Those lines might have different shapes and lengths but the existence of only one isolated line will lead to crushing and stopping of the processing. Before creating natural roads, one important step that has to be made is chopping of arcs at each junction, chopping of arcs at junctions is called building up topology and it can be made by using Data Interoperability Tool in ArcGIS. Natural roads are road segments connected to their adjacent one at the junction with the Gestalt principle for good continuation (Jiang et al., 2008). Axwoman extension in ARC GIS was used to create natural streets based on a predefined threshold angle. In this study a deflection angle of (45) degrees was used, in other words, intersected segments having deflection angle equal to or less than (45) degrees will be connected as one segment, the process will continue till the end of segments unless the condition is not fulfilled (Figure 3). The reason for choosing (45) degree as a threshold comes to the fact that If two road segments meet and angle between them is more than 45 then, most probably, they are two separate roads and are not one single continuous road.

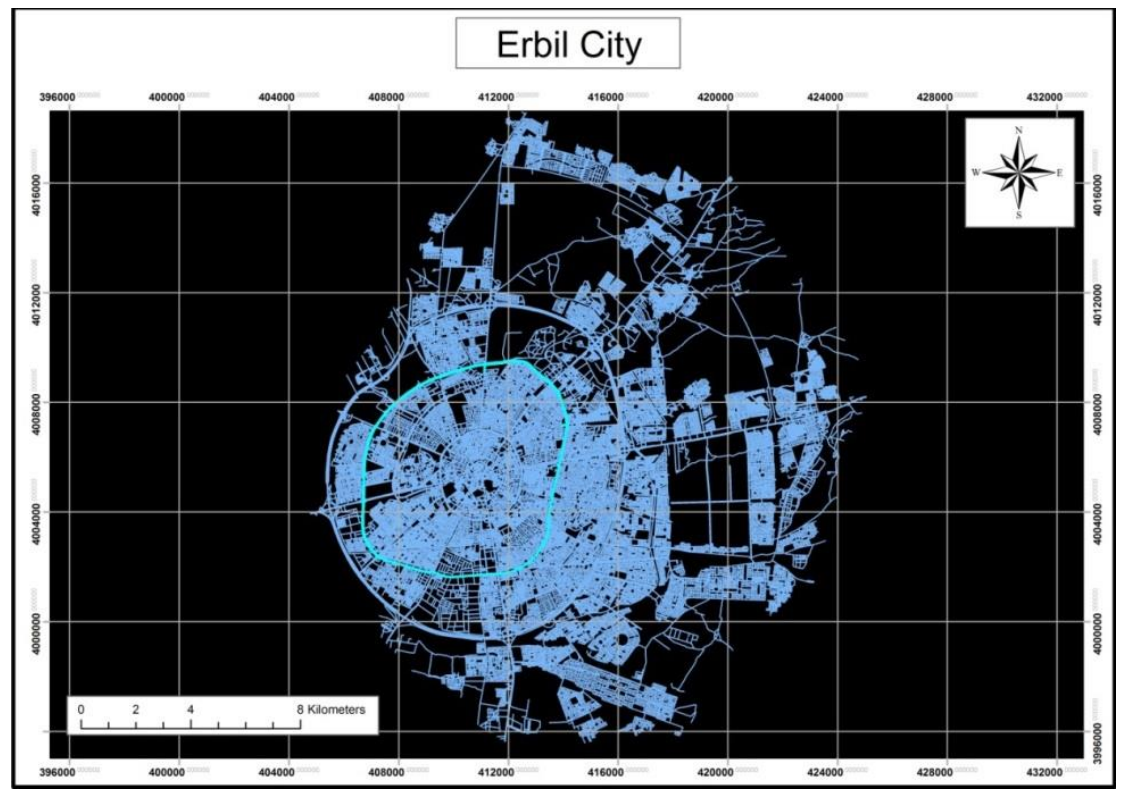

Figure 2. Creation of Natural Roads of a sample city (Erbil).

Next, MATLAB software was used to examine the scale-free property of street networks of each city. The degree distribution was checked to see if it fits a power-law distribution that follows the equation $\mathrm{y}=\mathrm{cx}-\mathrm{a}$. For the equation, $\mathrm{y}$ is the cumulative probability of occurrences of different degree $\mathrm{x}$. To check this property, two main steps must be followed:

1) Importing the connectivity value of each natural road which created in the previous step or creating a centrality value using Pajek software.

2) Three MATLAB codes must be used to get the results; first one was for finding the values of (alpha, xmin and D). The Second code is for the creation of a graph that shows the power-law as a straight line that follows a log-log graph. Third code is for finding how well the degree distribution follows a power-law distribution. A general rule of thumb is that if the value of $(p)$ is greater than zero then the condition will be fulfilled. A sample of the power law distribution graph is shown in (figure 4). Appendix A shows the power law distribution graphs for the all cities of concern in this study. 


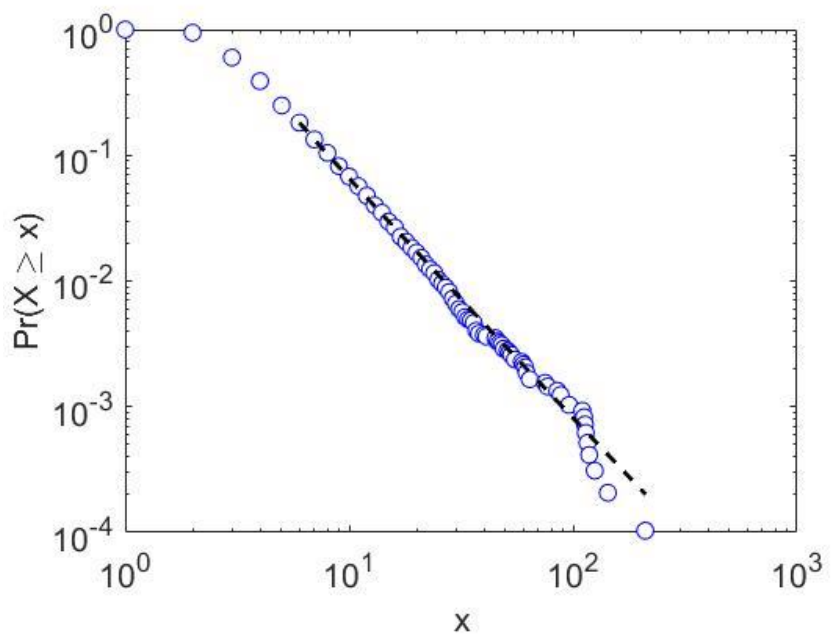

Figure 3. Power law distribution graph (Produced by MATLAB).

After this step, small world properties for each city were found. Urban streets or a network has small-world property when it has a small separation globally and highly clustered locally. In order to check whether the street network has a small-world property two measures must be found; average path length and clustering coefficient. Pajek software was used for this purpose and for comparing these two measures were calculated for a random graph which has the same degree per vertex and the same number of nodes. $\mathrm{L}$ random $=\mathrm{LN} \mathrm{n} / \mathrm{LN} \mathrm{m}, \mathrm{C} 1 \mathrm{random}=\mathrm{m} / \mathrm{n}$, where $\mathrm{m}$ is the mean value of a degree and $\mathrm{n}$ is the total number of vertices. An urban street network has a small-world property when $\mathrm{C} 1$ value is greater than $\mathrm{C} 1$ random.

In the final step Head/tail breaks classification was applied to reveal the hierarchical patterns within the street networks. Head/Tail Breaks is a new classification scheme in order to find groupings or hierarchy for data with a heavy-tailed distribution or as it is known as the data that has a scale-free property. The heavy-tailed distributions are heavily right skewed, with a minority of large values in the head and a majority of small values in the tail (Jiang, 2013). The classification is done by dividing things into large (or called the head) and small (or called the tail) things around the arithmetic mean or average, and then recursively going on for the division process for the large things or the head until the notion of far more small things than large ones is no longer valid, or with more or less similar things left only, which makes it significantly different from conventional methods of classification such as equal steps, quantiles, geometric progressions, standard deviation and natural breaks.

Small events are far more common in geographic spaces than large events. For example, there are far more small cities than large ones; far more short streets than long ones (Jiang, 2009); far more small city blocks than large ones, and far more low-density areas than high-density ones (Jiang \& Liu, 2011). Having said that, Head/tail breaks classification method is considered an effective classification method for most spatial data.

\section{Results and Discussions}

As mentioned previously our analysis based on urban streets in six cities, three large cities and three relatively small ones which have been divided into large-sized and small-sized groups. We intentionally chose cities with different sizes because urban planning strategies in large cities are different from smaller ones, the fact that larger cities contain more facilities and roads than small ones and having more population makes the demand on services to be higher. As a first step, the street data of each city was evaluated to see if the degree distribution fits power-law distribution depending on MATLAB software. The (P) value of each city was higher than zero which indicates that the data fits power-law distribution as it shown in (Table 1). MATLAB codes can also be used to plot the data on a graph to visually check whether the distribution of data fits power-law distribution. (Figure 5) is showing the plot of Kirkuk city as a sample. The results showed that all datasets are plotted as a straight line with a small deviation which confirms the power-law distribution property of each data set. After finishing this step, it is obvious that street networks in all cities have a scale-free property and their degree distribution fits a power-law distribution. 
Table 1. P-value of six cities.

\begin{tabular}{cc}
\hline City & P-Value \\
\hline Erbil & 0.618 \\
\hline Sulaumaniyah & 0.048 \\
\hline Kirkuk & 0.014 \\
\hline Duhok & 0.358 \\
\hline Halabja & 0.356 \\
\hline Koysinjaq & 0.664
\end{tabular}

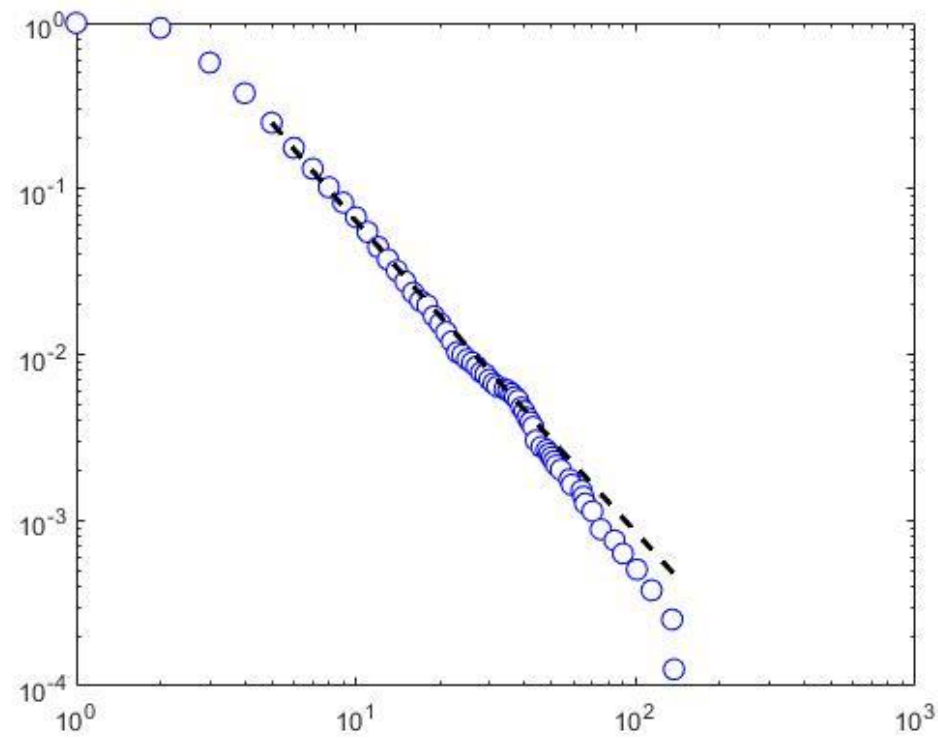

Figure 4. Street network of Kirkuk city follows power-law distribution.

Next, small-world properties of street networks of cities were analyzed. Average path length and clustering coefficient of all the cities were calculated as shown in (Table 2). The two measures for their random graphs were also calculated for comparison purposes.

Table 2. Small-World properties of street networks.

\begin{tabular}{lcccccc}
\hline City & \#segments & Mean Value & Clustering & Clustering $\boldsymbol{R}$ & $\begin{array}{c}\text { Avaerage } \\
\text { Path }\end{array}$ & $\begin{array}{c}\text { Avaerage } \\
\text { Path } \boldsymbol{R}\end{array}$ \\
\hline Erbil & 16912 & 4.3289 & 0.1345 & 0.0002 & 7.4780 & 6.8092 \\
\hline Sulaymaniyah & 7596 & 4.2304 & 0.1706 & 0.0006 & 6.5931 & 6.1953 \\
\hline Kirkuk & 7931 & 4.2330 & 0.1528 & 0.0005 & 6.5134 & 6.2225 \\
\hline Duhok & 4107 & 3.9221 & 0.1487 & 0.0010 & 7.1469 & 6.0883 \\
\hline Halabja & 1140 & 4.5175 & 0.1740 & 0.0040 & 5.7541 & 4.6677 \\
\hline Koysinjaq & 1167 & 3.8680 & 0.2164 & 0.0033 & 5.4759 & 5.2206 \\
\hline
\end{tabular}


As it can be noticed from the above table the highest degree of separation, indicated by average path length, is 7 which indicates a high connection between all networks within cities. In addition to that, the clustering values of all cities were bigger than the random clustering values. Based on these two evaluations it can be stated that all street networks of cities have a small-world property.

In previous steps the results of the analyses showed that the street networks of cities have a scale-free property, so the condition is fulfilled to apply Head/tail breaks classification on each set of data to uncover the hierarchy of street network for each city. By analyzing the results it is found that the highest hierarchical level, which is indicated by Ht index, was for to Kirkuk city which belongs to a large-cities group having a value of 7, whereas the minimum hierarchical level could be seen for Halabja city that belongs to a smallcities group having a value of 4. Table (3) showing Ht_index value of each city, and table (4 and 5) showing a sample of the Head/tail classification details for a small and a big city that are (Halabja and Kirkuk) respectively. Appendix B demonstrates the Head/tail statistics for all cities in this study.

Table 3. Ht_index value for all cities

\begin{tabular}{cc}
\hline City & P-Value \\
\hline Erbil & 6 \\
\hline Sulaumaniyah & 7 \\
\hline Kirkuk & 8 \\
\hline Duhok & 6 \\
\hline Halabja & 4 \\
\hline Koysinjaq & 7 \\
\hline
\end{tabular}

Table 4. Head/tail breaks classification details for Halabja city

\begin{tabular}{cccccc}
\hline \multicolumn{7}{c}{ Halabja City } \\
\hline \#Data & \#head & \%head & \#tail & \%tail & Mean \\
\hline 1140 & 335 & $29 \%$ & 805 & $71 \%$ & 4.52 \\
\hline 335 & 110 & $32 \%$ & 225 & $68 \%$ & 9.49 \\
\hline 110 & 32 & $29 \%$ & 78 & $71 \%$ & 15.6 \\
\hline
\end{tabular}

Table 5. Head/tail breaks classification details for Kirkuk city

\begin{tabular}{cccccc}
\hline \multicolumn{7}{c}{ Kirkuk City } \\
\hline \#Data & \#head & \%head & \#tail & \%tail & Mean \\
\hline 7931 & 1984 & $25 \%$ & 5947 & $75 \%$ & 4.23 \\
\hline 1984 & 534 & $26 \%$ & 1450 & $74 \%$ & 9.39 \\
\hline 534 & 135 & $25 \%$ & 399 & $75 \%$ & 18.01 \\
\hline 135 & 48 & $35 \%$ & 87 & $65 \%$ & 34.36 \\
\hline 48 & 14 & $29 \%$ & 34 & $71 \%$ & 54.73 \\
\hline 14 & 5 & $35 \%$ & 9 & $65 \%$ & 84.86 \\
\hline 5 & 2 & $40 \%$ & 3 & $60 \%$ & 115.4 \\
\hline
\end{tabular}


After applying the Head/tail break classification to street networks of cities, the hierarchical patterns could be shown by using ArcGIS software with Axwoman extension by calculating space syntax parameters. In the produced maps, streets with the highest connectivity were represented with red color and streets with lowest connectivity were represented with dark blue color. Streets which have high connectivity are very few in comparison to those which have less connectivity. This minority part of streets play an important role in human life and research showed that the $20 \%$ of top streets accommodate a majority of traffic flow (Jiang, 2009). Highly connected streets tend to attract more movement of people and vehicles than low connected ones. Moreover, it can be stated that the streets of highest connectivity and are most important in the network where most activities within the city will be performed like social, financial, economic and industrial activities. This hierarchical representation provides a perceived way for urban planners and engineers to better understand the city's morphology. Figures (6 and 7) showing the map of both Halabja and Kirkuk cities after calculating space syntax parameters using GIS with Axwoman extension.

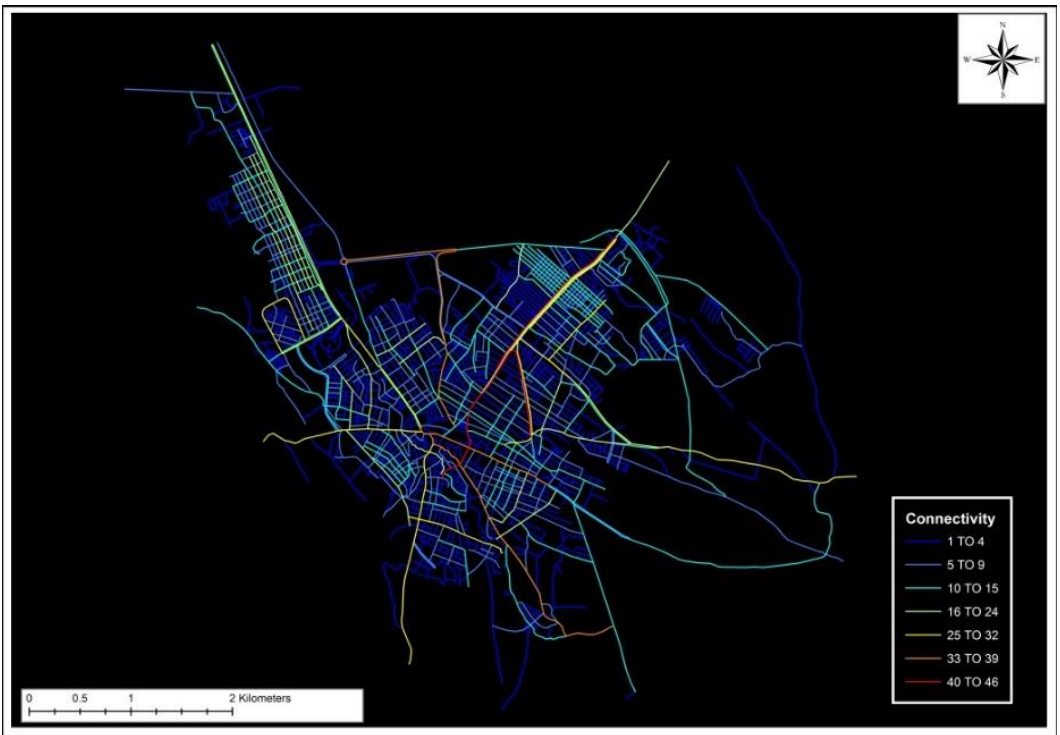

Figure 6. Map of Halabja city.

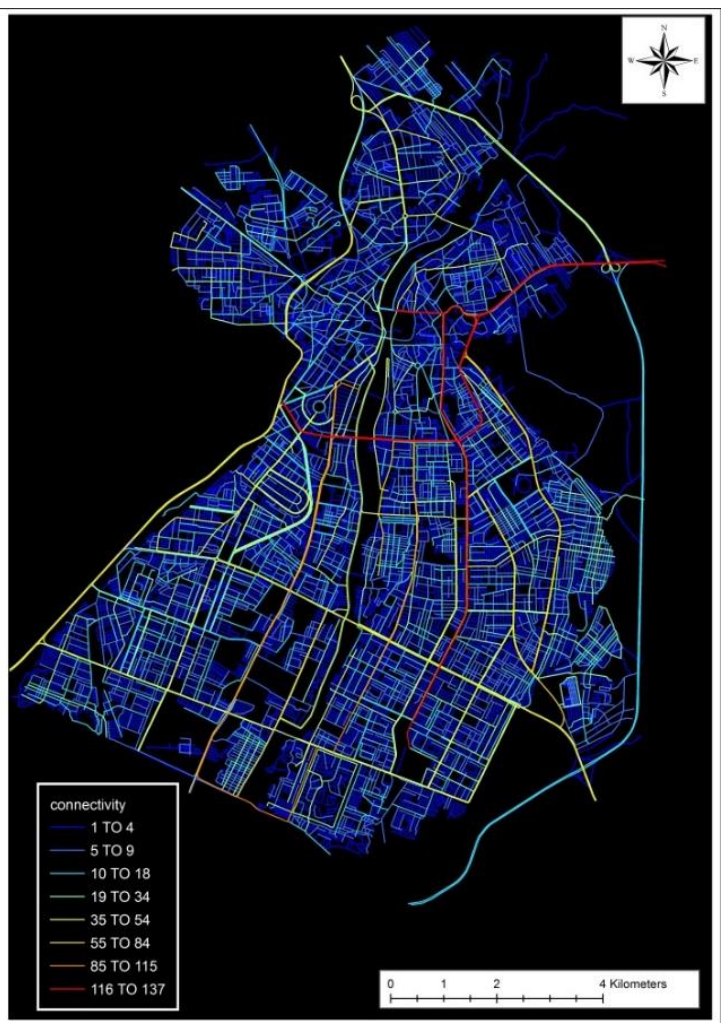

Figure 7. Map of Kirkuk city.

As can be realized from both figures, red lines which represent streets with the the highest connectivity are very few comparing with the blue lines representing low connectivity streets. A universal street structure states that $80 \%$ of streets have the the connectivity of less than four (average connectivity of streets), while $20 \%$ of streets have connectivity greater than the average (Jiang, 2007). The streets with the the highest connectivity are considered to be the the most important ones in the network. It could be seen that streets with the the highest connectivity were a very small part of the network and it supported the idea that vital streets are the the minority and few streets can form a backbone of the network (Jiang, 2007). Urban planners and engineers can use such map to understand the hierarchical pattern of streets within the city, this approach enables the authorities to make decisions towards making cities more sustainable. Cities which have a a high hierarchical level, represented by Ht_index value, tend to be more beautiful and living than those having low Ht_index value. Following the street structure, over $80 \%$ of traffic occurred in the $20 \%$ of well-connected streets; while only $20 \%$ of traffic occurred in the $80 \%$ less connected streets (Jiang, 2009). These are all crucial data that can be considered in future urban planning decisions. 


\section{Conclusion}

The purpose of this study was to provide a different point of view towards urban planning of cities. To test the methodology presented in this paper to optimum, 6 different cities were chosen for analysis, 3 big cities and 3 relatively small ones. The motivation behind choosing cities of different sizes is the fact that street structure in big cities is varies from small ones. Moreover, the number of streets within large cities is also higher, this property causes streets in large cities to have more connections than those in small cities. Furthermore, urban planning in large cities is different from that in small cities because the demands of services are higher in large ones. The basic idea of this kind of analysis is to provide helpful tools for specialized people in making better strategic planning decisions in large and small cities.

Statistical analysis computed for this study showed that all the cities had a scale-free property or the data of their street networks were heavily tailed distribution; a majority of streets having connections less than average while the the minority of streets have connections more than average. In addition to that, street networks of both groups of large-sized cities and small-sized ones had the small-world properties after evaluating the average path length and clustering property of each individual city. Applying the Head/tail breaks classification to heavily distributed street networks of cities showed that; the highest hierarchical level value was found in a large-sized city having Ht-index value of (8), and the small-sized city had the lowest hierarchical level having Ht-index value of only (4). The value of the hierarchical level of other cities gathered between (4 and 8).

The basic idea of this kind of analyses is to provide a different point of view in urban planning. By using the results of these analyses, specialized people in urban planning are able to make better strategic planning decisions in large cities. Moreover, it also can be used for making minor decisions like modifying the locations of public service facilities into more suitable locations. Critical new buildings such as (hospitals, firefighting stations, police stations, etc.) can be planned in spots in such a way that easy access is guaranteed for citizens. Urban planner can use the produced maps and analysis to immediately perceive that which streets have most connectivity in a city, which areas tend to attract most people, and which areas within the city are calmer than other parts. Moreover, the analyses also show which parts of city need to be developed to make it more attractive, where next school, hospital, shopping center or any other public service building should be built depending on the hierarchical pattern of streets inside cities.

Finally, the ultimate goals of urban planning are to make cities more living, sustainable and beautiful. The analyses done in this study provide helpful tools towards understanding the street structure of cities, the maps produced by this analysis is not like conventional city maps that only show the geometric property of features but in contrast it also shows the topological characteristics and the relations of features in the map. It is a powerful tool to help in designing more efficient and living cities. The results can be beneficial in making crucial decisions in urban planning.

\section{References}

Jianquan C. and Masser I (2001), Towards a Spatial Analysis Framework: Modelling Urban Development Patterns. Geocomputation Conference Brisbane.

Goodchild M. F. (2007), Citizens as Sensors: the World of Volunteered Geography. GeoJournal, 69(4), $211-221$.

Goodchild M. F , May Yuan \& Cova T. J. (2007), Towards a general theory of geographic representation in GIS, International Journal of Geographical Information Science, 21(3), 239-260 .

Haklay M. (2010). How good is Volunteered Geographical Information? A comparative study of OpenStreetMap and Ordnance Survey datasets. Environment and Planning B:Planning and Design,37(4), 682-703.

Haklay M. and Weber P. (2008). OpenStreetMap: User-Generated Street Maps. Pervasive Computing, IEEE, 7(4), 12-18.

Hillier B. (2009), The Now and the Future of Space Syntax: From structures and models to theory, keynote speech, $9^{\text {th }}$ International Space Syntax Symposium Seoul.

Hillier B. and Hanson J. (1984), The Social Logic of Space, Cambridge University Press:Cambridge.

Hiller B., Leaman A., Stansall P., and Bedford M. (1976). Space Syntax, Environment and Planning B: Planning and Design. 1976;3(2):147-185. doi:10.1068/b030147 
Hillier B. and Sahbaz O. (2005), High resolution analysis of crime patterns in urban streetnetworks: an initial statistical sketch from an ongoing study of a London borough, proceedings of $5^{\text {th }}$ International Space Syntax Symposium Delft, $451-478$.

Hussain M.R. (2016). "An Overview of Geographic Information System (GIS)". 10.13140/RG.2.1.3569.5603.

Jiang B. (2007). A Topological Pattern of Urban Street Networks: Universality and Peculiarity. Physica A:Statistical Mechanics and its Application, 384(2), 647-655.

Jiang B. (2009), Street hierarchies: a minority of streets account for a majority of traffic flow, International Journal of Geographical Information Science, 23(8), 1033-1048.

Jiang B. (2013). Head/tail breaks: A new classification scheme for data with a heavy-tailed distribution. The Professional Geographer, 65(3), 482-494.

Jiang B. (2018), A Topological Representation for Taking Cities as a Coherent Whole. Geogr Anal, 50(3), $298-313$.

Jiang B. and Claramunt C. (2002), A structural approach to model generalization of an urban street Network, Proceedings of the $5^{\text {th }}$ A GILE Conference on Geographic Information Science, Mallorca, Spain, 25-27 April 2002, $205-217$.

Jiang B and Claramunt C. (2002), Integration of space syntax in to GIS: New perspective for urban morphology, Blackwell Publisher Ltd, 108 Cowley road, Oxford OX4 1JF, UK and 350 main street, malden, MA 02148, USA, 6(3), $295-309$.

Jiang B and Claramunt C (2004), Topological analysis of urban street networks, Environment and Planning B: Planning and Design, 31(1), 151-162

Jiang B. and Liu C. (2009) Street-based topological representations and analyses for predicting traffic flow in GIS, International Journal of Geographical Information Science, 23(9),1119-1137.

Jiang B. and Liu X. (2011), Scaling of geographic space from the perspective of city and field blocks and using volunteered geographic information, International journal of geographic information science, 26(2),215-229.

Jiang B., Zhao S. and Yin j. (2008), Self-Organized Natural Roads for Predicting Traffic Flow: A Sensitivity Study, Journal of Statistical Mechanics: Theory and Experiment P07008.

LeGates R.T., Tate N.J., and Kingston R. (2009), Spatial thinking and scientific urban planning, Environment and Planning B: Planning and Design, volume 36, pages 763-768.

Mitzenmacher M. (2004), A brief history of generative models for power law and lognormal distributions, Internet Mathematics, $1(2), 226-251$.

Murayama Y. and Thapa R.B. (2011). Spatial Analysis and Modeling in Geographical 1 Transformation Process, The GeoJournal Library 100, DOI 10.1007/978-94-007-0671-2_1

Thomson R.C. (2003), Bending the axial line: smoothly continuous road centre-line segments as a basis for road network analysis. In Proceedings of the Fourth Space Syntax International Symposium, University College London, London J. Hanson (Ed.).

Yuan M., Mark M. D., Egenhofer J. M. and Peuquet J. D. (2005), Extensions to Geographic Representations, A Research Agenda for Geographic Information Science, 129-56, edited by McMaster B. R. and Usery L. E., New York: CRC Press.

Watts, D. J. and Strogatz, S. H. (1998), Collective dynamics of "small-world" networks, Nature, 393, 440-442. 


\section{Appendix A}

Figures (A1-A6) showing the power-law distribution property of street network of the selected cities.

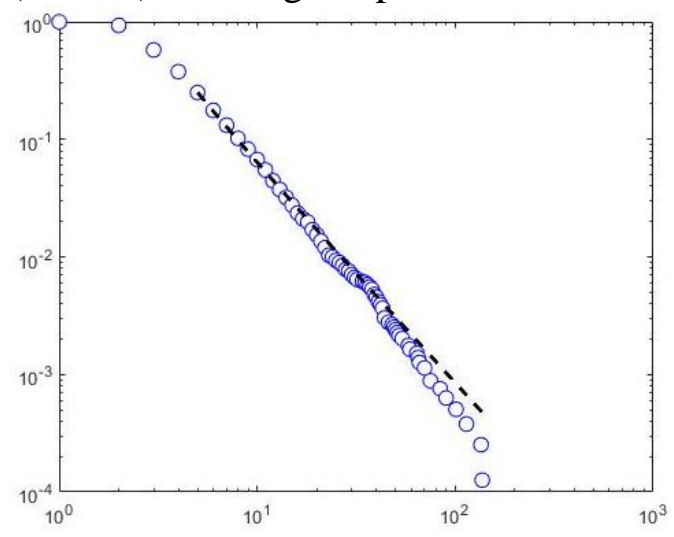

Figure A1. Erbil City

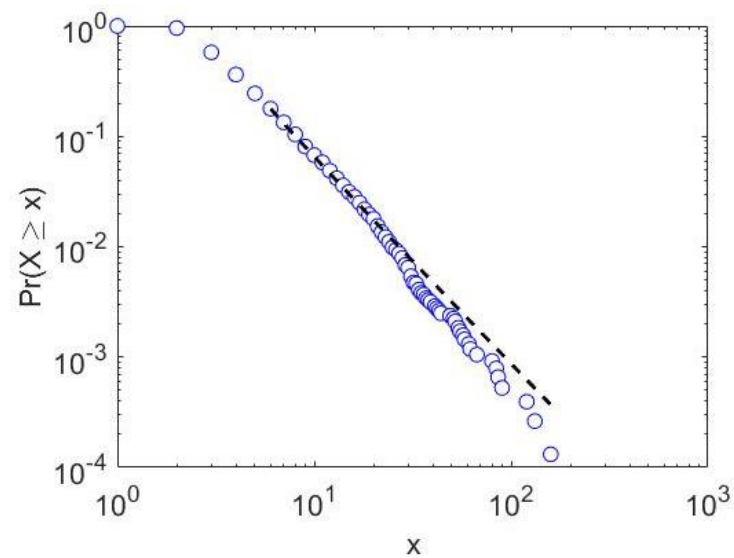

Figure A3. Sulaymaniyah City

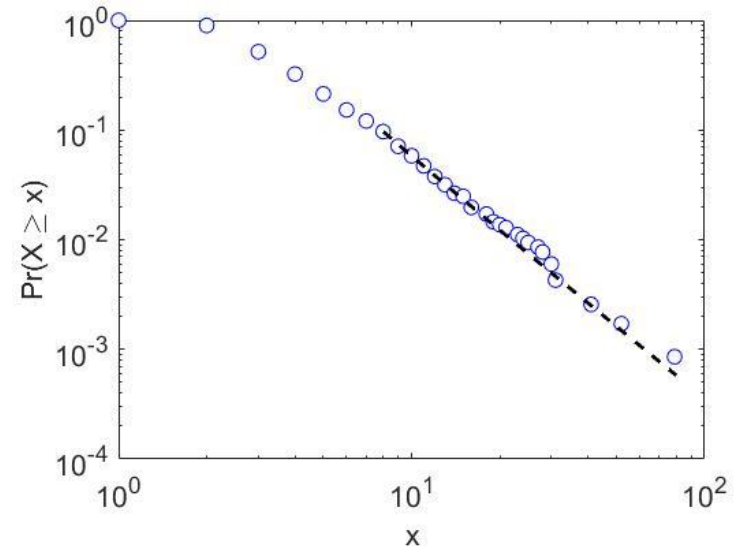

Figure A5. Koysinjaq City

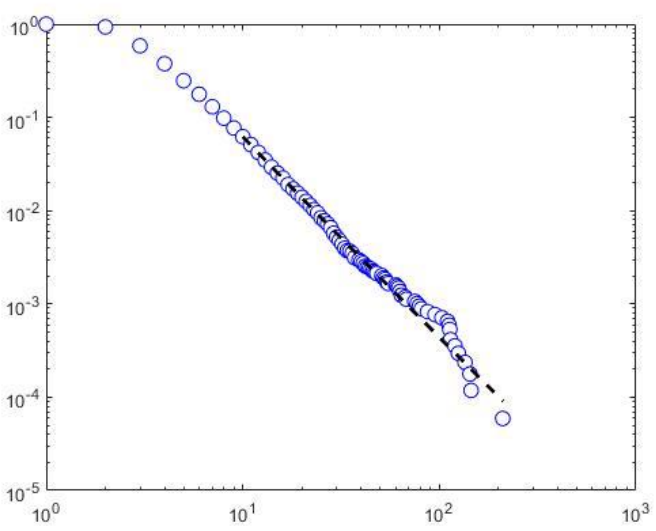

Figure A2. Kirkuk City

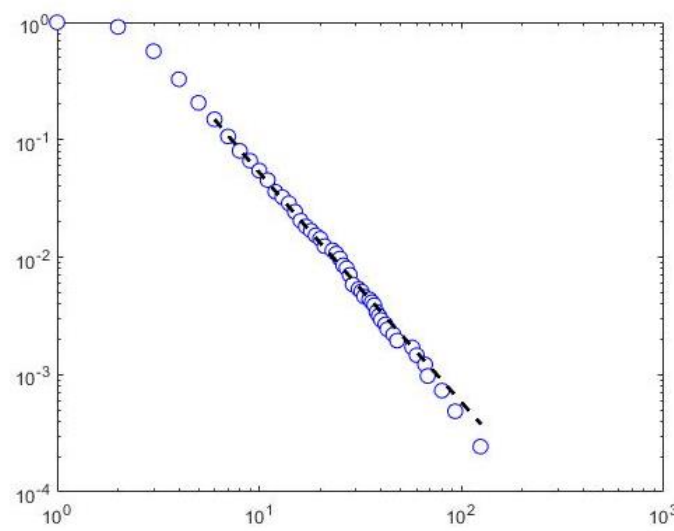

Figure A4. Duhok City

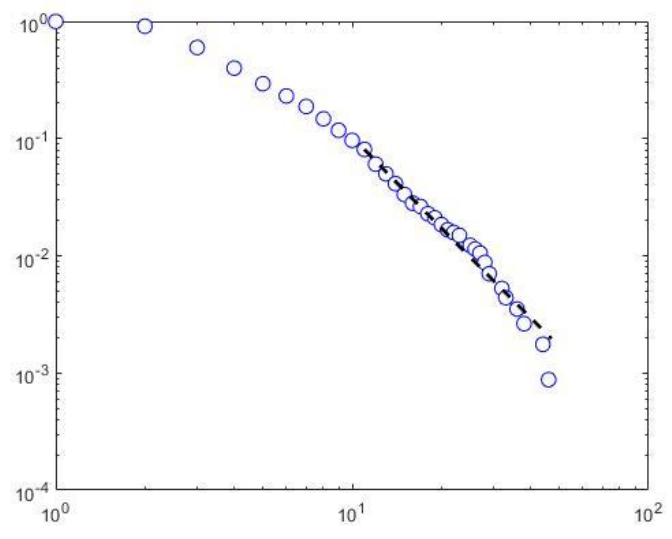

Figure A6. Halabja City 


\section{Appendix B}

Statistical Results of Head/tail Breaks classification are shown in tables (B1-B6). (Note: \# $=$ number, $\%=$ percentage),

Table B1. Statistical results of Head/tail breaks classification of Erbil City

\begin{tabular}{|c|c|c|c|c|c|}
\hline \multicolumn{7}{|c|}{ Erbil } \\
\hline \#Data & \#head & \%head & \#tail & \%tail & mean \\
\hline 16912 & 4183 & $24 \%$ & 12729 & $76 \%$ & 4.18 \\
\hline 4183 & 1050 & $25 \%$ & 3133 & $75 \%$ & 9.18 \\
\hline 1050 & 290 & $27 \%$ & 760 & $73 \%$ & 17.89 \\
\hline 290 & 67 & $23 \%$ & 223 & $77 \%$ & 32.49 \\
\hline 67 & 21 & $31 \%$ & 46 & $69 \%$ & 63.63 \\
\hline
\end{tabular}

Table B3. Statistical results of Head/tail breaks classification of Sulaymaniyah City

\begin{tabular}{|c|c|c|c|c|c|}
\hline \multicolumn{7}{|c|}{ Sulaymaniyah } \\
\hline \#Data & \#head & $\%$ head & \#tail & \%tail & mean \\
\hline 7596 & 1861 & $24 \%$ & 5735 & $76 \%$ & 4.26 \\
\hline 1861 & 513 & $27 \%$ & 1348 & $73 \%$ & 9.57 \\
\hline 513 & 149 & $29 \%$ & 364 & $71 \%$ & 18.23 \\
\hline 149 & 36 & $24 \%$ & 113 & $76 \%$ & 31.5 \\
\hline 36 & 12 & $33 \%$ & 24 & $67 \%$ & 56.42 \\
\hline 12 & 4 & $33 \%$ & 8 & $67 \%$ & 88 \\
\hline
\end{tabular}

Table B5. Statistical results of Head/tail breaks classification of Koysinjaq City

\begin{tabular}{|c|c|c|c|c|c|}
\hline \multicolumn{7}{|c|}{ Koysinjaq } \\
\hline \#Data & \#head & $\%$ head & \#tail & \%tail & mean \\
\hline 1167 & 378 & $32 \%$ & 789 & $68 \%$ & 3.87 \\
\hline 378 & 113 & $29 \%$ & 265 & $71 \%$ & 7.48 \\
\hline 113 & 31 & $27 \%$ & 82 & $73 \%$ & 13.61 \\
\hline 31 & 11 & $35 \%$ & 20 & $65 \%$ & 24.19 \\
\hline 11 & 3 & $27 \%$ & 8 & $73 \%$ & 36.55 \\
\hline
\end{tabular}

Table B2. Statistical results of Head/tail breaks classification of Kirkuk City

\begin{tabular}{|c|c|c|c|c|c|}
\hline \multicolumn{7}{|c|}{ Kirkuk } \\
\hline \#Data & \#head & \%head & \#tail & $\%$ tail & mean \\
\hline 7931 & 1984 & $25 \%$ & 5947 & $75 \%$ & 4.23 \\
\hline 1984 & 534 & $26 \%$ & 1450 & $74 \%$ & 9.39 \\
\hline 534 & 135 & $25 \%$ & 399 & $75 \%$ & 18.01 \\
\hline 135 & 48 & $35 \%$ & 87 & $65 \%$ & 34.36 \\
\hline 48 & 14 & $29 \%$ & 34 & $71 \%$ & 54.73 \\
\hline 14 & 5 & $35 \%$ & 9 & $65 \%$ & 84.86 \\
\hline 5 & 2 & $40 \%$ & 3 & $60 \%$ & 115.4 \\
\hline
\end{tabular}

Table B4. Statistical results of Head/tail breaks classification of Duhok City

\begin{tabular}{|c|c|c|c|c|c|}
\hline \multicolumn{6}{|c|}{ Duhok } \\
\hline \#Data & \#head & \%head & \#tail & \%tail & mean \\
\hline 4107 & 1342 & $32 \%$ & 2765 & $68 \%$ & 3.92 \\
\hline 1342 & 330 & $24 \%$ & 1012 & $76 \%$ & 7.42 \\
\hline 330 & 84 & $25 \%$ & 246 & $75 \%$ & 15.15 \\
\hline 84 & 22 & $26 \%$ & 62 & $74 \%$ & 29.04 \\
\hline 22 & 7 & $31 \%$ & 15 & $69 \%$ & 50.77 \\
\hline
\end{tabular}

Table B6. Statistical results of Head/tail breaks classification of Halabja City

\begin{tabular}{|c|c|c|c|c|c|}
\hline \multicolumn{7}{|c|}{ Halabja } \\
\hline \#Data & \#head & \%head & \#tail & \%tail & mean \\
\hline 1140 & 335 & $29 \%$ & 805 & $71 \%$ & 4.52 \\
\hline 335 & 110 & $32 \%$ & 225 & $68 \%$ & 9.49 \\
\hline 110 & 32 & $29 \%$ & 78 & $71 \%$ & 15.6 \\
\hline
\end{tabular}




\section{Appendix C}

The hierarchical level representations of street networks of all cities are shown in figures (C1-C6).

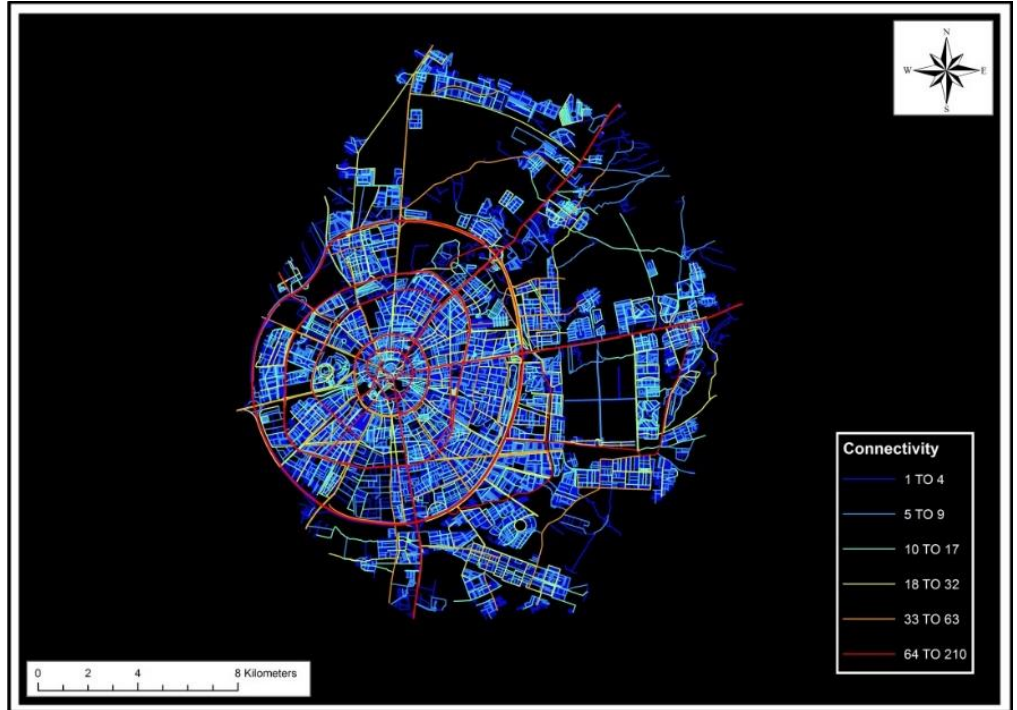

Figure C1. Erbil city

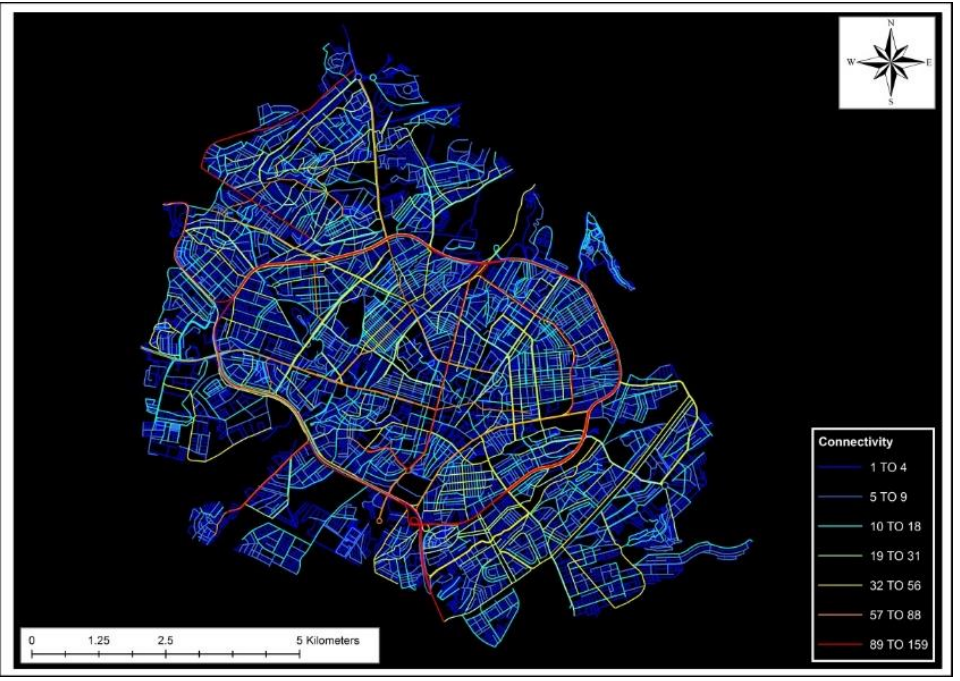

Figure C3. Sulaymaniyah city

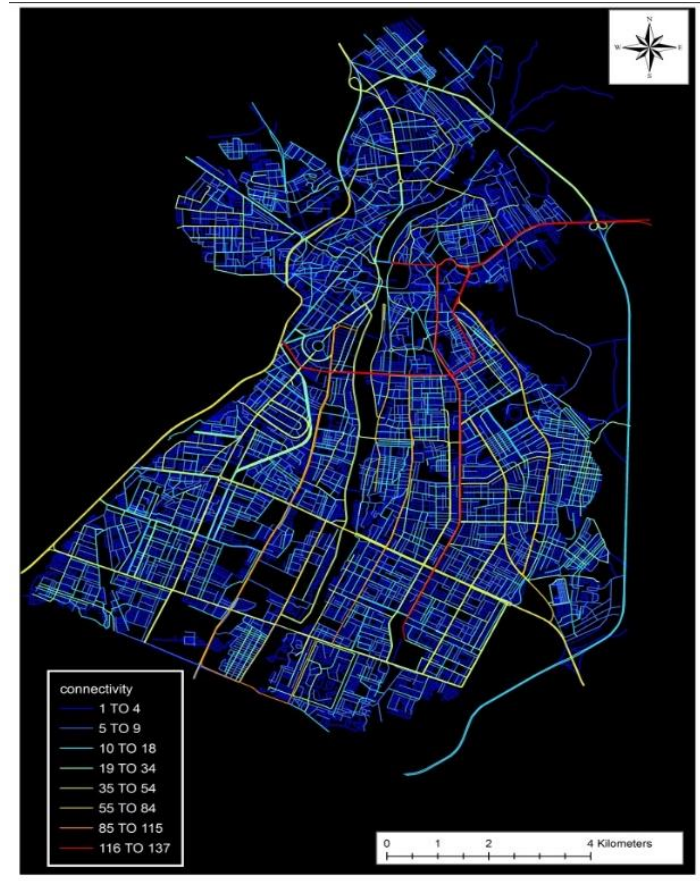

Figure C2. Kirkuk city

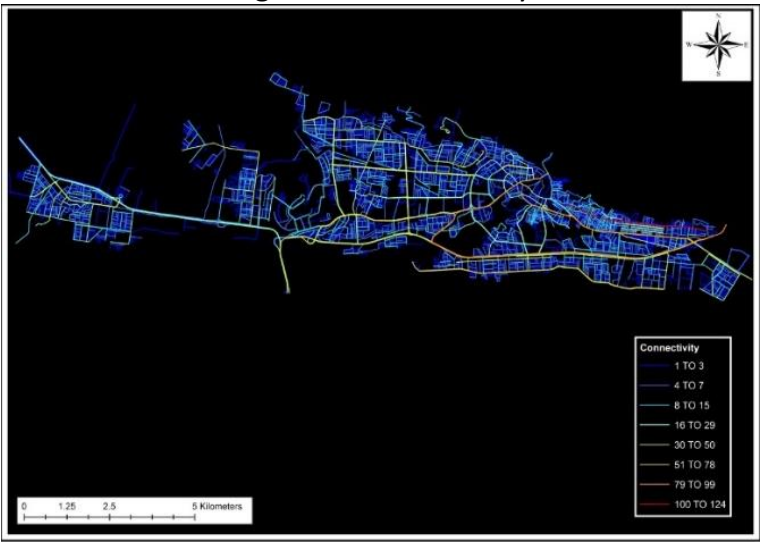

Figure C4. Duhok city 


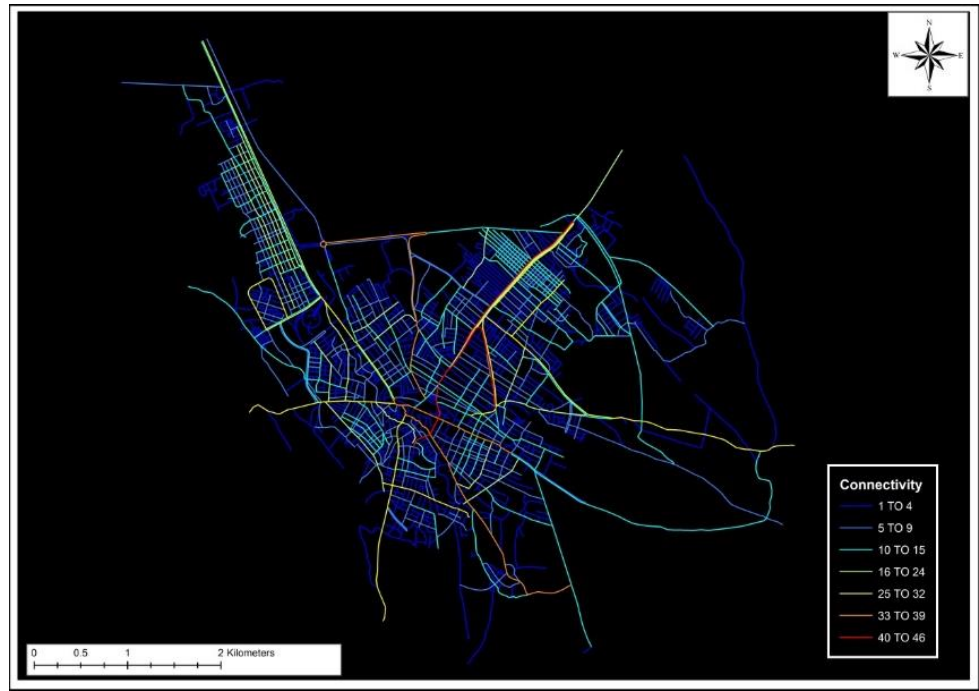

Figure C6. Halabja city

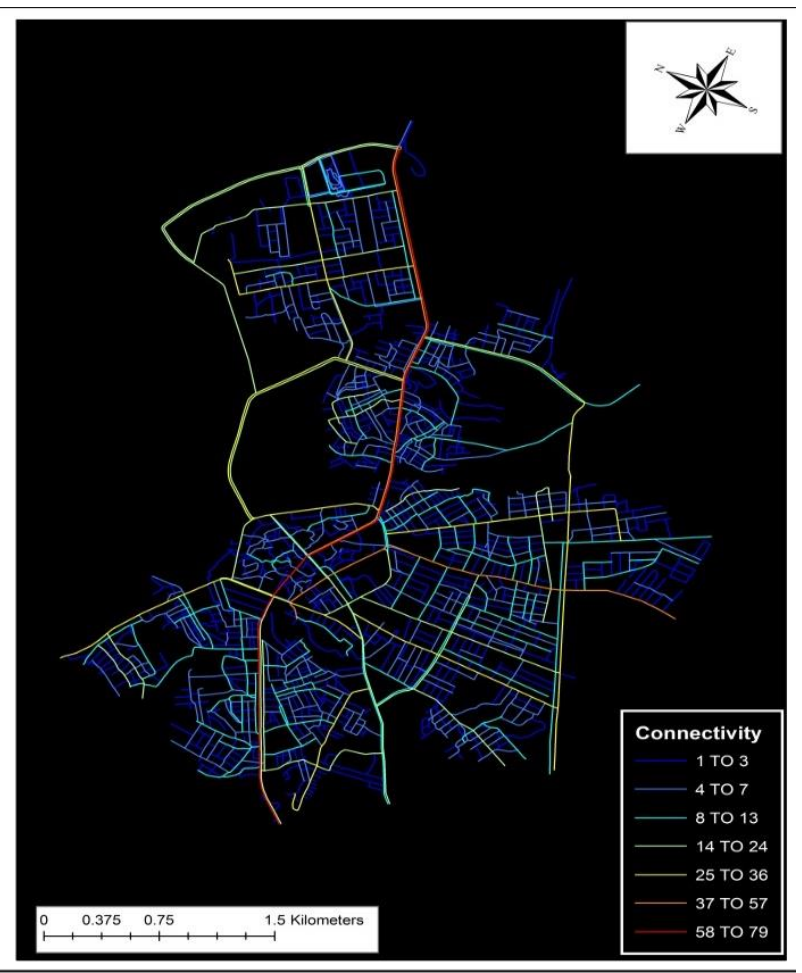

Figure C5. Koysinjaq city 